Chrischta Ganz

\section{Das Leben von Pfarrer Sebastian Kneipp}

Sebastian Kneipp (Abb. 1) wurde am 17. Mai 1821 in eine arme Weberfamilie in Stephansried bei Ottobeuren im bayrischen Allgäu geboren. Zusammen mit seinen vier Schwestern erlebte er eine karge, strenge und freudlose Kindheit. Neben der Schule halfen alle fünf Kinder am Webstuhl mit, den Unterhalt der Familie sicherzustellen. Als Jugendlicher und junger Mann arbeitete Kneipp hart als Knecht, Maurer oder Tagelöhner, um seinen Traum, das Priesterstudium, zu verwirklichen. 1842 - Kneipp war 21 Jahre alt - brannte das Haus seiner Familie in Stephansried nieder, sodass die Familie Kneipp alles verlor. Tatkräftig half Sebastian Kneipp beim Wiederaufbau und der Versorgung der Familie mit. Einige Monate später wurde er von Kaplan Merkle auf das Studium vorbereitet. Seinen Lebensunterhalt verdiente er weiterhin mit schwerer körperlicher Arbeit als Knecht. Mit 23 Jahren konnte Kneipp das Gymnasium in Dillingen beginnen. Dort erkrankte er an Tuberkulose, an der er die ganze Schulzeit litt. Als 28-Jähriger begann er in äusserst schlechter körperlicher Verfassung das Theologiestudium in München. Sein Zustand verschlechterte sich weiter. 2 Jahre später gaben ihn die Ärzte auf; Kneipp war todkrank. In der Hofbibliothek fand Kneipp die Aufzeichnungen von Johann Siegmund Hahn: «Von der Kraft und der Wirkung des frischen Wassers». Mit letzter Kraft begann Kneipp, dreimal wöchentlich im kalten Fluss kurze Tauchbäder zu nehmen. Dies war der Wendepunkt in seinem Gesundheitszustand. Später schrieb er darüber:

\title{
Die Kneipp'sche Gesundheitslehre
}

Pfarrer Sebastian Kneipp entwickelte im 19. Jahrhundert eine Gesundheitslehre, die auf Wasseranwendungen, volksheilkundlichem Wissen, Erfahrungsheilkunde und Lebensordnung basiert. Kneipp ist heute einer der bekanntesten Vertreter der Hydrotherapie.

«Müde ging ich hinaus, neu aufgefrischt und gestärkt ging ich heim und gewann die Überzeugung, wenn es für mich ein Heilmittel gibt, so wird es das Wasser sein» [1].

Mit den Wasseranwendungen verbesserte sich sein Gesundheitszustand langsam, aber stetig.

1852 erhielt Kneipp, vollständig genesen, die Priesterweihe und kam als Kaplan nach Biberbach, Boos bei Memmingen, Augsburg und schlussendlich nach Wörishofen. Als 1854 mit der ersten deutschen Industrieausstellung in München eine Choleraepidemie ausbrach, behandelte er erfolgreich viele Cholera-Patienten und erhielt den Beinamen "CholeraKaplan».

In Wörishofen leitete er neben seinen kirchlichen Aufgaben die Landwirtschaft und richtete eine Kräuterapotheke ein. Viele Kranke, auch prominente Patienten, suchten ihn auf und gesundeten mit seiner Therapie. Seine wachsende Bekanntheit schürte jedoch den Neid und die Missgunst vieler Mediziner. Einige Ärzte aus der Region begannen aber trotzdem, mit Kneipp zusammenzuarbeiten.

Zwischen 1886 und 1889 schrieb Kneipp seine Bücher «Meine Wasserkur» und «So sollt ihr leben», die, in viele Sprachen übersetzt, weltweit erfolgreich waren.

Der Kneippverein Wörishofen wurde gegründet, es erschienen die ersten «Kneipp-Blätter» und Kneipp verfasste den «Ratgeber zur Kinderpflege». Viele Vortragsreisen folgten.

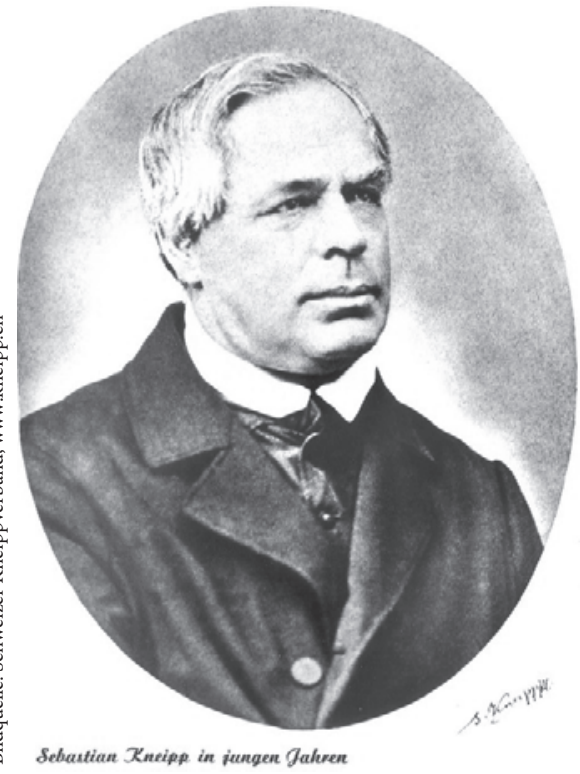

Abb. 1. Sebastian Kneipp in jungen Jahren ( ${ }^{(}$Familie Grebmer).

Weltweit wurden über 100 KneippKurhäuser sowie der Internationale Kneippverbund gegründet (mit über 70 Vereinen und 10000 Mitgliedern). Wörishofen wurde zum offiziellen Kneippkurort mit ärztlich geführtem Sanatorium.

1897 verstarb Kneipp als bekannter und hoch geschätzter Mann in Wörishofen $[1,2]$.

\section{Die Kneipp'sche \\ Gesundheitslehre}

Kneipps Lehre vom gesunden Leben und der Naturheilkunde überzeugt in ihrer Einfachheit, Natürlichkeit und

\section{KARGER}

(C) 2016 S. Karger GmbH, Freiburg 
Heilwirkung. Seine Gesundheitslehre ist ein ganzheitliches Lebenskonzept ohne aufsehenerregende oder kostspielige Behandlung von Symptomen. Seine Empfehlungen basieren auf einer den Naturgesetzen folgenden Lebenshaltung und fordern vom einzelnen Menschen ein hohes Mass an Eigenverantwortung. Die Kneipp'sche Gesundheitslehre wird sowohl zur Prävention (Kneipp spricht von «Gesunderhaltung» [1]) als auch zur Behandlung von Kranken und in der Rekonvaleszenz angewendet. Grundprinzipien seiner Therapie sind aktive Immunstärkung, Abhärtungsmassnahmen, Erholung (Regeneration) und Kräftigung [1,2].

Sicherlich ist die Hydrotherapie der bekannteste Teil der Gesundheitslehre von Pfarrer Sebastian Kneipp. Dennoch werden an dieser Stelle alle fünf Säulen seiner Lehre kurz vorgestellt, denn die fünf Teilaspekte ergänzen und unterstützen einander und ergeben ein ganzheitliches Therapiekonzept $[1,2]$.

(1) Kräuterkunde: Kneipp verordnete sowohl die innerliche Gabe von Heilpflanzen als auch die äusserliche Anwendung von einheimischen Heilpflanzen und folgte damit der jahrtausendealten Tradition der Heilpflanzenkunde [1, 2]. «Unser Herrgott hat für jedes Leiden ein Kräutlein wachsen lassen» (Sebastian Kneipp [1]).

(2) Ernährung: Kneipp empfahl eine ausgewogene faserstoffreiche Vollwertkost aus frischen und biologischen Lebensmitteln mit einem hohen Rohkostanteil. Raffinierte und denaturierte Nahrungsmittel sollen reduziert bzw. gemieden werden $[1,2]$.

«Die Zubereitung der Speisen soll einfach und ungekünstelt sein. Je näher sie dem Zustande kommen, in welcher sie von der Natur geboren werden, desto gesünder sind sie» (Sebastian Kneipp [1]).

(3) Bewegung: Die Kneipp'sche Bewegungstherapie beinhaltet sowohl aktive als auch passive Bewe- gung, soll mit Freude ausgeübt werden und soll der Konstitution des Einzelnen angepasst sein. Bewegung an der frischen Luft wird bevorzugt und kann gut mit den Wasseranwendungen kombiniert werden $[1,2]$.

«Untätigkeit schwächt, Übung stärkt, Überlastung schadet» (Sebastian Kneipp [1]).

(4) Innere Ordnung: Von allen fünf Säulen ist der Bereich der Ordnungslehre und Lebensordnung, wie die innere Ordnung auch genannt wird, sicherlich der anspruchsvollste. Kneipp verstand darunter ein Leben in Einklang mit den Gesetzen der Natur, eine lebensbejahende innere Haltung und positives Handeln im Alltag. Der Mensch solle im Leben den richtigen Rhythmus finden sowie die verschiedenen Lebensaspekte massvoll leben. Damit gemeint ist ein Gleichgewicht zwischen Aktivität und Regeneration, Wachen und Schlafen und allen anderen Lebensäusserungen. Eine ökologische Grundhaltung mit sinnvoller Nutzung von Licht, Luft, Wasser, Erde, Nahrung usw. sowie Nächstenliebe und Hilfsbereitschaft sind für Kneipp ebenso in die innere Ordnung eingebettet $[1,2]$.

(5) Wasseranwendungen: Die Wasseranwendungen sind, wie bereits erwähnt, die bekannteste Säule der Kneipp'schen Gesundheitslehre. Sie umfassen Güsse, Waschungen, Bäder und Wickel. Alle menschlichen Grundfunktionen wie Kreislauf, Atmung, Wärmehaushalt, Immunsystem und die Stoffwechselprozesse werden durch hydrotherapeutische Reize reguliert. Das Wasser überträgt die thermischen, mechanischen und chemischen Reize auf den menschlichen Körper und veranlasst ihn zu gesundheitsfördernden Reaktionen [1].

"Ist das Wasser für den gesunden Menschen ein vorzügliches Mittel, seine Gesundheit und Kraft zu er- halten, so ist es auch in der Krankheit das erste Heilmittel, Es ist das natürlichste, einfachste, wohlfeilste und, wenn recht angewendet, das sicherste Mittel» (Sebastian Kneipp [1]).

\section{Hydrotherapie}

Allgemein werden unter dem Begriff Hydrotherapie Wasseranwendungen zur Prävention und in der Therapie verstanden. Die Anwendungen zu Heilzwecken wurden schon im Altertum praktiziert und gehören unter anderem zu den wesentlichen Therapieformen der Traditionellen Europäischen Naturheilkunde (TEN).

Die Kneipp'sche Wassertherapie basiert auf dem Wissen von Kneipps Vorgängern Vincenz Priessnitz, Johann Siegmund Hahn und Eucharius Ferdinand Christian Örtel. Sebastian Kneipp hat die Wasseranwendungen aufgrund seiner Beobachtungen und Erfahrungen weiter verfeinert und optimiert.

\section{Reize setzen}

Die Art, Intensität, Temperatur und Dauer des Reizes bestimmt die Wirkung der hydrotherapeutischen Anwendung. Die Reizstärke wird von den Faktoren Temperatur, Dosierungszeit, Grösse des Areals der gereizten Fläche (Erwärmungsorte) und zusätzlichen Einflussfaktoren wie biochemische, phytotherapeutische wie auch mechanische Reize (z.B. Bürstenbäder) beeinflusst [1-3].

\section{Reaktion am Beispiel eines Kaltwasserreizes}

Ein Kaltwasserreiz löst eine Engstellung der Gefässe aus, was sich in blasser Haut oder Gänsehaut zeigt. Der Reiz wird von den Nervenzellen der Haut zum Zentralnervensystem und danach zum vegetativen Nervensystem weitergeleitet. Der Kaltwasser- 
Tab. 1. Regeln der Hydrotherapie [1,2]
Kaltwasserreize regulieren und stabilisieren den Wärmehaushalt.

Warme Wasseranwendungen, vor allem als Bäder, wirken hauptsächlich beruhigend.

Wechselwarme Anwendungen sind induziert bei schlechtem Reaktionsvermögen und wenig belastbarer Konstitution.

Nach einer Kneipp-Anwendung muss man sich wohlfühlen. Das ist das klare Zeichen einer gesunden Reaktion auf eine richtig durchgeführte und angepasste Anwendung.

Keine kalten Wasser- oder Wickelanwendungen bei einem kalten Körper oder bei Untergewicht.

Nach jeder Kaltanwendung auf Wiedererwärmung achten.

Erwärmung der Körperteile durch langsam ansteigende Temperatur und Anwendungen von Wasser. Ausnahme nach Kneipp zur Erlangung von warmen Gliedmassen: Einreibung der kalten Füsse und Hände mit Schnee.

Bei jeder Wasseranwendung den Zustand des Patienten beachten.

Keine Regel ohne Ausnahme, die Therapie ist an den jeweiligen Zustand des Patienten anzupassen.

Bei Körpersensationen wie Übelkeit, Kopfschmerzen, Herzrasen und Übermüdung sind starke Reize zu vermeiden.

Je chronischer die Erkrankung, desto sanfter (und längerfristiger) muss der Reiz der Wasseranwendung sein.

Wassertherapeutische Anwendungen müssen regelmässig durchgeführt, der Situation angepasst trainiert und gesteigert werden (Disziplin und Achtsamkeit).

reiz aktiviert die Makrophagen und beeinflusst die T- und B-Zell-Regulation. Die Zunahme von sekretorischem Immunglobulin A und Gamma-Interferon löst nicht nur eine Durchblutungsförderung der Haut und Schleimhäute, sondern auch eine positive Beeinflussung zentralnervöser Leistungen aus [3].

\section{Wirkung}

Nach der Engstellung der Gefässe durch den Kältereiz erweitern sich diese, was die Hautdurchblutung verbessert und reflektorisch auf die inneren Organe wirkt. Die Organleistung, Zellernährung und Verwertung des Sauerstoffs werden verbessert, die Lymphtätigkeit und die Stoffwechselfunktionen gesteigert und Stoffwechselendprodukte vermehrt ausgeschieden. Auch das Herz-Kreislauf-System wird entlastet. Ziel der Kneipp'schen Wasseranwendungen ist die «Stabilisierung und Harmonisierung der Grundfunktionen wie Atmung, Kreislauf, Wärmehaushalt, Stoffwechsel, Verdauung, Nerven- und Hormonsystem sowie die Aktivierung und Stär- kung des Immunsystems und damit eine gefestigte Gesundheit» (Tab. 1) [1].

\section{Wassertreten}

Das Wassertreten ist die älteste Form der Kneipp-Anwendungen. In der Badewanne oder einem Tretbecken wird gerade so viel kaltes Leitungswasser eingefüllt, bis es zur Wade reicht. Abwechselnd werden im Storchengang (Abb. 2) die Füsse und Unterschenkel aus dem Wasser gehoben und wieder eingetaucht. Nach 20-60 s wird das Wasser nur abgestreift, die Haut aber nicht getrocknet. Danach soll der Patient schnell gehen, barfuss über Steine oder Waldboden laufen oder warme Socken anziehen, um die Füsse zu erwärmen. Wassertreten wirkt ausgleichend: Am Morgen aktiviert und am Abend beruhigt es [1,2].

\section{Tautreten}

Eine weitere Form der Abhärtung ist das morgendliche Tautreten. Mit noch bettwarmen Füssen wird die feuchte, mit Tau bedeckte Wiese im Storchengang betreten. Dabei werden

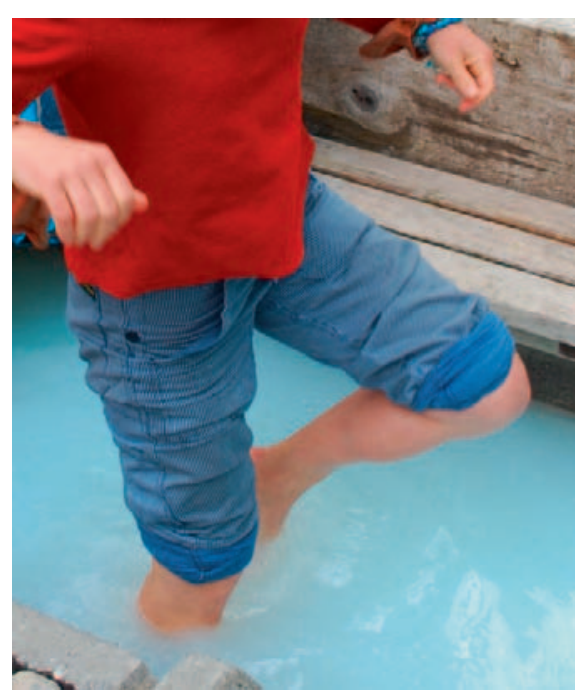

Abb. 2. Storchengang.

die Stoffwechselvorgänge am Morgen sanft angeregt. Danach soll der Patient, auch hier ohne die Füsse abzutrocknen, warme Socken anziehen, um sie wieder zu erwärmen. Tautreten wirkt kräftigend, kreislaufanregend, durchblutungsfördernd, venenkräftigend, immunstärkend, vegetativ stabilisierend und abhärtend.

\section{Schneelaufen}

Der Patient/die Patientin geht barfuss über weichen Schnee, sollte aber nie stehen bleiben. Kurz vor dem Kälteschmerz werden die Füsse kurz abfrottiert und in Socken gepackt. Die Wirkung ist dem Tautreten ähnlich, aber intensiver.

\section{Bäder und Dämpfe}

Bäder werden als Teil-, Sitz- und Vollbad angewendet, sowohl mit konstanter als auch sich verändernder Temperatur oder im Wechsel zwischen einem kalten und einem warmen Bad (wechselwarme Bäder). Sie setzen je nach Art, der individuellen Reaktionslage und der Dauer einen anderen Reiz. Oft fügt man den Bädern verschiedene Kräuter hinzu, um die Wirkung gezielt zu verstärken (Abb. 3, 4). Für Dämpfe wird heisses Wasser mit Kräuterzusätzen eingesetzt (Tab. 2). 
Tab. 2. Verschiedene Bäder [2]

\begin{tabular}{llll}
\hline Art des Bades & Dauer & Temperatur, ${ }^{\circ} \mathrm{C}$ & Besonderes \\
\hline Überwärmungsbad & $3-10 \mathrm{~min}$ & $40-43$ & bewirkt Mehrdurchblutung \\
Ansteigendes Bad & $10-15 \mathrm{~min}$ & $33-40$ & langsamer Anstieg \\
Wechselbäder & $5 \mathrm{~min}$ warm, & 38, & beginnen mit warm, beenden \\
& 10 min kalt & 18 (Leitungswasser) & $\begin{array}{l}\text { mit kalt oder warm je nach } \\
\text { Konstitution und Beschwerden }\end{array}$ \\
& $10 \mathrm{~min}$ & 38 & $\begin{array}{l}\text { starke Mehrdurchblutung } \\
\text { Warmes Bad }\end{array}$ \\
Temperiertes Bad & $10-20 \mathrm{~s}$ & $16-22$ & sanfte Mehrdurchblutung \\
Kaltes Bad & $5-10 \mathrm{~s}$ & 18 (Leitungswasser) & anregend \\
\hline
\end{tabular}

Abb. 3. Armbad (Foto ${ }^{\circledR}$ Schweizer Kneippverband, www.kneipp.ch).

Abb. 4. WechselFussbad (Foto ${ }^{\circledR}$ Schweizer Kneippverband, www.kneipp.ch).

Abb. 5. Knieguss (Illustration: Rolf Stickel; mit freundlicher Genehmigung aus [1], S. 88; ${ }^{\circledR}$ Schweizer Kneippverband).
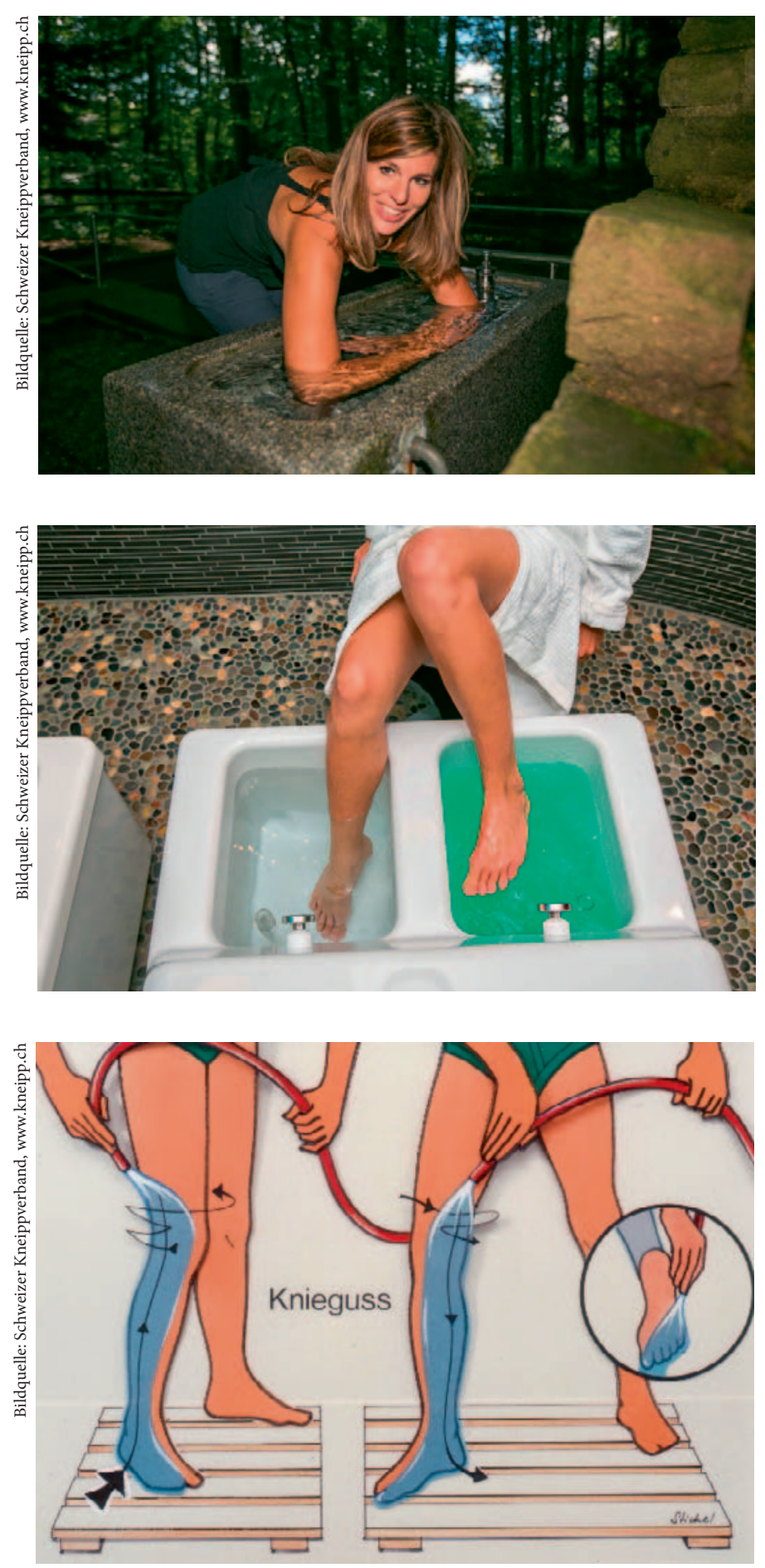

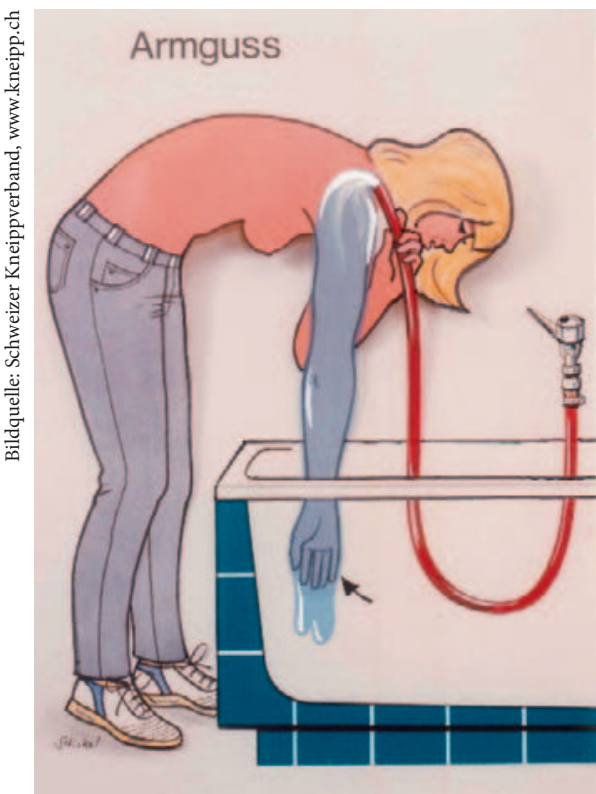

Abb. 6. Armguss (Illustration: Rolf Stickel; mit freundlicher Genehmigung aus [1], S. 83; ${ }^{\circ}$ Schweizer Kneippverband).

\section{Waschungen}

Mit einem Waschlappen wird ein dünner, gleichmässiger Wasserfilm auf den gewählten Körperteil oder auf den ganzen Körper aufgetragen. Wichtig ist, dabei zügig, aber ohne Hast zu arbeiten. Kalte Waschungen zählen zu den mildesten der Kneipp'schen Wasseranwendungen. Sie wirken abhärtend, beruhigend und nervenstärkend, verbessern die Hautdurchblutung, entlasten das Herz-Kreislauf-System, stabilisieren den Wärmehaushalt, regen den Stoffwechsel und die Ausscheidung an und harmonisieren das vegetative Nervensystem.

\section{Güsse}

Abgüsse wurden ursprünglich mit der Giesskanne durchgeführt; heute verwendet man im häuslichen Bereich einen speziellen Schlauch mit grossem Ausguss. Schläuche für Kneipp'sche Güsse sind auch in vielen Thermalbädern sowie in der Sauna und in Dampfbädern anzutreffen. Die Kneipp'schen Güsse sind ein charakteristischer Bestandteil der Kneipp'schen Hydrotherapie, wurden von Sebastian Kneipp 


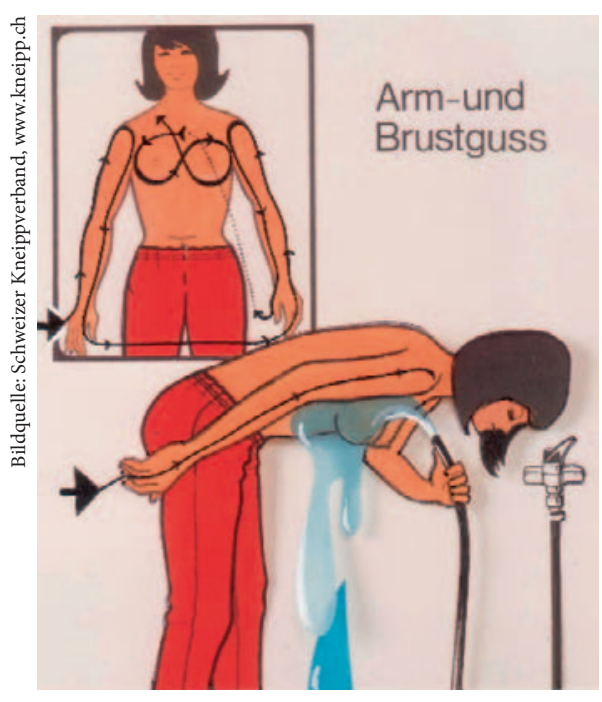

Abb. 7. Arm- und Brustguss (Illustration: Rolf Stickel; mit freundlicher Genehmigung aus [1], S. 85, (c) Schweizer Kneippverband). selbst entwickelt und sind auch nach ihm benannt.

Bei einem Guss wird ein druckloser, gleichmässig fliessender Wasserstrahl in einem bestimmten Ablauf auf die Haut aufgetragen, der den betroffenen Körperteil in einem Wassermantel weich umspült. Begonnen wird immer an der herzfernsten Stelle.

Der Temperaturreiz eines Gusses regt als Reaktion die aktive Durchblutung stark an und aktiviert damit die gesamte Wärmeregulation. Über die Haut wirken Güsse auf den Kreislauf, das Nervensystem, die Ausscheidung und den Stoffwechsel (Abb. 5-7).

\section{Wickel und Kompressen}

Wickel und Kompressen sind altbewährte Heilanwendungen aus der
Volks- und Erfahrungsmedizin, die einen sanften Reiz setzen und gleichzeitig sehr effektiv wirken. Ein Wickel umhüllt mit einem nassen und zwei trockenen Tüchern einen Körperteil (z.B. Wadenwickel). Kompressen und Auflagen wirken lokal am gewählten Ort. Sowohl Wickel als auch Kompressen und Auflagen wirken durch einen Temperaturreiz, können aber auch mit Heilpflanzenzusätzen gezielt verstärkt werden.

\section{Literatur}

1 Meier R: Praktische Kneipp-Anwendungen. Bern, Schweizer Kneippverband, 2000.

2 Raimann C, Ganz C, Garvelmann F, BertschiStahl HD, Fehr-Streule R: Grundlagen der Traditionellen Europäischen Naturheilkunde. Schiedlberg, Bacopa, 2012.

3 Süssmuth A: Kneipp'sche Wasserkur. Naturheilpraxis 3/2016;16-20. 